\title{
Estrategia de educación y comunicación ambiental para el manejo integral de residuos orgánicos en la colonia zipor, Xalapa, ver.
}

\section{Environmental education and communication strategy for the integrated management of organic waste in the zipor neighborhood, Xalapa, Veracruz.}

DOI: $10.46932 / \mathrm{sfjdv2n2-200}$

Received in: March 1st, 2021

Accepted in: May 30th, 2021

\author{
Ana Karen Ricaño Ochoa \\ Maestra en G.A.S. \\ RCTI de México, S. de R. L. de C.V. Km38.5 Carretera 57Querétaro-San Luis Potosí, San José \\ Iturbide, Guanajuato, México
}

\author{
Dra. María de los Ángeles Chamorro Zárate achamorro \\ Universidad Veracruzana. Lomas del estadio s/n, Zona Universitaria, Xalapa, Ver., México
}

\begin{abstract}
RESUMEN
Este proyecto de la Maestría en Gestión Ambiental para la Sustentabilidad se aplicó en la Colonia Zipor, en la ciudad de Xalapa, Veracruz, México. Esta ciudad ha pasado por algunos problemas de gestión de residuos, incluido el aumento de la generación de residuos, el cierre del vertedero de la ciudad y los residuos importados. Los objetivos de este proyecto fueron: diseñar y aplicar acciones de educación y comunicación ambiental para lograr la sensibilización y formación en materia de residuos: promover la participación ciudadana en el proceso de gestión de residuos, especialmente en la clasificación y tratamiento biológico de los residuos orgánicos: instalar huertos urbanos comunitarios para su aprovechamiento el compost producido durante el tratamiento biológico.

Se trabajó con tres grupos de diez vecinos cada uno, que representó el 5\% de los habitantes de esta zona. El proyecto tuvo tres etapas, que se aplicaron a cada grupo. La primera etapa consistió en la enseñanza de la separación de residuos, la segunda etapa fue la construcción de módulos de compost y la capacitación para su manejo, finalmente, la tercera etapa consistió en la instalación de huertos urbanos. Se impartieron dos talleres de separación de residuos, se construyeron dos módulos de compost y se hicieron los preparativos para el primer huerto urbano con la ayuda de la Red de Agricultura Urbana de Xalapa.

Para aplicar este proyecto y lograr las metas fue muy importante la colaboración e involucramiento de la Dirección de Medio Ambiente y Sustentabilidad de Xalapa, la sociedad y la academia.
\end{abstract}

Palabras clave: gestión de residuos, participación ciudadana, educación ambiental

\begin{abstract}
This project from the Master in Environmental Management for Sustainability applied in the Colonia Zipor, in the city of Xalapa, Veracruz, México. This city has gone though some problems of waste management, including the increase of waste generation, the closure of the city landfill and imported waste. The objectives of this project they were design and apply environmental education and communication actions to achieve awareness and training in waste matter: promote citizen engagement in the waste management process, especially in classifying and giving biological treatment to organic waste: install communitarian urban gardens to utilize the compost produced during the biological treatment.

It worked with three groups of ten neighbors each one, what did it represent $5 \%$ of the habitants of this zone. The project had three stages, that were applied to every group. The first stage consisted of the teaching of waste separation, the second stage was the construction of compost modules and training for
\end{abstract}


its management, finally, the third stage consisted of the installation of urban gardens. Two waste separation workshops were held, two compost modules were built, and preparations were made for the first urban garden with the help of the Xalapa Urban Agriculture Network.

To apply this project and achieve the goals, the collaboration and involvement of the Xalapa Environment and Sustainability Directorate, society and academia was very important.

Keywords: waste management, citizen engagement, environmental education.

\section{INTRODUCCIÓN}

La estrategia de educación y comunicación ambiental se realizó en el marco del Trabajo Recepcional de la Maestría en Gestión Ambiental para la Sustentabilidad (MGAS) de la Universidad Veracruzana. Los ejes principales son la participación ciudadana, el manejo integral de Residuos Sólidos Urbnaos (RSU) y la vinculación entre gobierno-ciudadanía-academia. La estrategia se implementó para atender el problema en materia de residuos que enfrenta el municipio de Xalapa, derivado del aumento en la generación de basura, el descontento social con el servicio de limpia pública, el cierre del relleno sanitario El Tronconal y la inadecuada gestión de RSU.

Las actividades se llevaron a cabo en la Colonia Zipor, donde en enero de 2018 se aplicó un Plan Piloto de Recolección Diferenciada y a partir del $1^{\circ}$ de octubre del mismo año inició oficialmente la entregra de los RSU separados al camión recolector. A través de la participación ciudadana se están fortaleciendo la separación de RSU desde la fuente y se promueve el aprovechamiento de los residuos orgánicos en compostas y huertos comunitarios. La vinculación entre los diferentes actores involucrados ha permitido la realización de este proyecto con éxito y con miras a un manejo integral de los residuos.

El presente trabajo tuvo como objetivos

- Diseñar acciones de educación y comunicación ambiental para sensibilizar y capacitar a los ciudadanos en materia de manejo integral de residuos orgánicos.

- Promover la participación ciudadana en los procesos de separación de RSU y en el tratamiento biológico de los residuos orgánicos.

- Instalar huertos urbanos comunitarios para el aprovechamiento del abono obtenido en el compostaje.

\section{METODOLOGÍA}

Para realizar el presente trabajo, se integró una metodología conformad por seis etapas, tomando como referencia elementos contenidos en los trabajos realizados por Ruíz, 2009; Meza, 2010; Robles et 
al., 2010; Aguilera, 2016; Hernández, 2014; Vaquerizo, 2015; Applus+, 2016 y Martín \& Urquiaga, 2016. Las seis etapas son consecutivas y la etapa de comunicación se aplicó transversalmente.

\subsection{ETAPA TRANSVERSAL: ESTRATEGIA DE COMUNICACIÓN}

En la estrategia de comunicación se utilizaron medios impresos, auditivos y digitales para sensibilizar a los participantes en materia de RSU, así como para difundir el proyecto e informar sobre las reuniones y resultados. Se diseñaron volantes, carteles, infografías y spots (para perifoneo). También se creó una página de Facebook y un sitio web, ambos llamados Eco Líderes Zipor. En esta etapa se involucró la Dirección de Participación Ciudadana, para apoyar en la difusión del proyecto, las reuniones y los talleres casa por casa.

\section{Etapa 1: Revisión Bibliográfica}

En un inicio se hizo una revisión bibliográfica para conocer la situación actual de RSU en Xalapa, Ver., el problema que se presenta, sus causas y los actores involucrados.

\section{Etapa 2: Vinculación}

Se estableció la vinculación con las autoridades municipales, a través de la Dirección de Medio Ambiente y Sustentabilidad, la Dirección de Participación Ciudadana y con los habitantes de la Colonia Zipor.

\section{Etapa 3: Recorrido por la Colonia Zipor y Selección de las Áreas Verdes}

Se realizaron recorridos por la Colonia Zipor con el equipo del Departamento de Agroecología y Educación Ambiental de la Subdirección de Recursos Naturales y Cambio Climático (perteneciente a la Dirección de Medio Ambiente y Sustentabilidad) para conocer los espacios disponibles para aplicar la estrategia. Se eligieron tres áreas verdes, las cuales se presentan en la figura 1. 
Figura 1: Áreas verdes seleccionadas para la aplicación de la estrategia de Educación y Comunicación

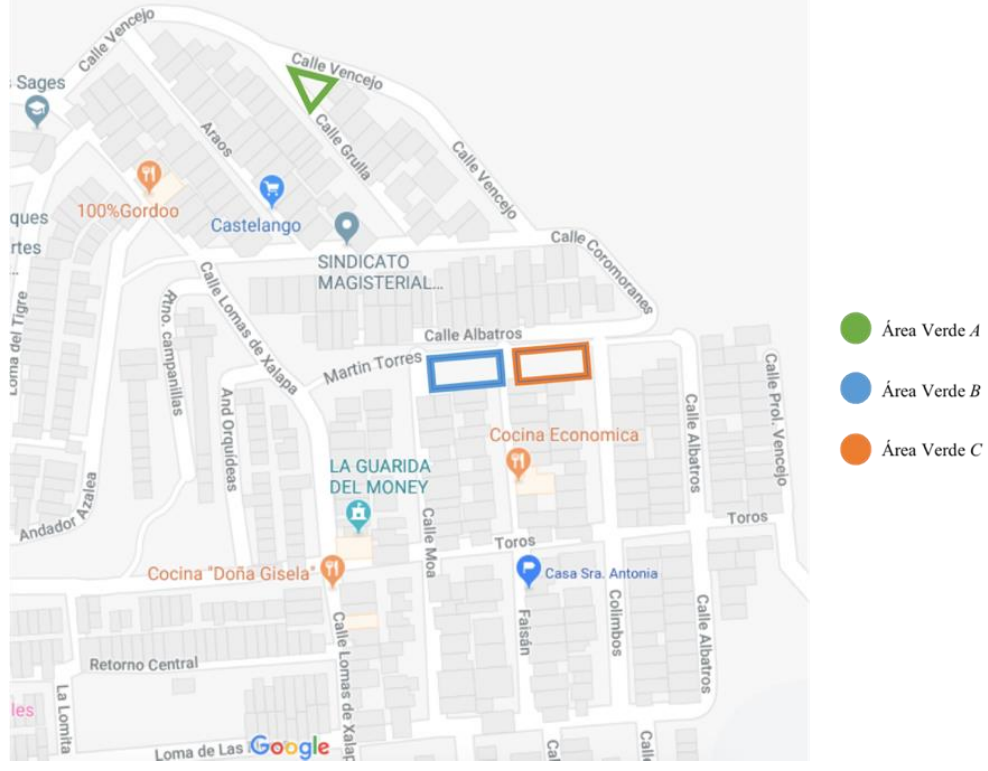

Etapa 4: Capacitación para la Separación de Residuos Sólidos Urbanos

La cuarta etapa correspondió a la aplicación de talleres de capacitación para separar los RSU y para que los participantes identificaran los problemas generados por el manejo inadecuado de los RSU, así como la importancia de su participación para separarlos. Los talleres de capacitación fueron diseñados y facilitados por el Departamento de Agroecología y Educación Ambiental. En la tabla 1 se presentan las acciones que se realizaron, así como los insumos y la aportación de los distintos actores involucrados.

Tabla 1 Acciones, Insumos y Vinculaciones de la Etapa 4

\begin{tabular}{|c|c|c|c|}
\hline Acción & Insumos & Tipo de Aportación & Vinculación \\
\hline $\begin{array}{l}\text { Gestión del taller en la } \\
\text { Subdirección de Recursos } \\
\text { Naturales y Cambio } \\
\text { Climático }\end{array}$ & - & Gestión & $\begin{array}{l}\text { Academia (Universidad } \\
\text { Veracruzana/MGAS) }\end{array}$ \\
\hline $\begin{array}{l}\text { Gestión de un espacio } \\
\text { para impartir el taller }\end{array}$ & Casa & Especie & $\begin{array}{c}\text { Vecinos de la Colonia } \\
\text { Zipor }\end{array}$ \\
\hline Impartición del taller & $\begin{array}{l}\text { Proyector, computadora y } \\
\text { material de apoyo (botes } \\
\text { de basura color naranja, } \\
\text { verde y gris y residuos) }\end{array}$ & $\begin{array}{c}\text { Especie/ } \\
\text { Recursos Humanos }\end{array}$ & $\begin{array}{c}\text { Departamento de } \\
\text { Agroecología y } \\
\text { Educación Ambiental }\end{array}$ \\
\hline
\end{tabular}

Nota: elaboración propia.

\section{Etapa 5: Construcción del Módulo de Compostaje y Capacitación para su Manejo}

En la quinta etapa se desarrollaron dos sesiones. En la sesión A se construyó el módulo de compostaje comunitario y en la Sesión B se capacitó a los vecinos sobre su manejo, ver tabla 2. 
Tabla 2 Acciones, Insumos y Vinculaciones de la Etapa 5

\begin{tabular}{|c|c|c|c|c|}
\hline Sesión & Acción & Insumos & $\begin{array}{c}\text { Tipo de } \\
\text { Aportación }\end{array}$ & Vinculación \\
\hline \multirow[b]{2}{*}{ A } & $\begin{array}{c}\text { Compra de } \\
\text { materiales para la } \\
\text { construcción del } \\
\text { módulo de } \\
\text { compostaje }\end{array}$ & $\begin{array}{c}\text { Tarimas de madera, malla } \\
\text { gallinera, plástico, clavos, } \\
\text { engrapadora, martillo, } \\
\text { pinzas, taladro }\end{array}$ & Especie & $\begin{array}{c}\text { Departamento de } \\
\text { Agroecología y } \\
\text { Educación Ambiental }\end{array}$ \\
\hline & $\begin{array}{l}\text { Construcción de } \\
\text { módulo de } \\
\text { compostaje }\end{array}$ & $\begin{array}{c}\text { Tarimas de madera, malla } \\
\text { gallinera, } \\
\text { plástico/Herramientas }\end{array}$ & $\begin{array}{l}\text { Recursos } \\
\text { Humanos }\end{array}$ & $\begin{array}{c}\text { Departamento de } \\
\text { Agroecología y } \\
\text { Educación Ambiental } \\
\text { Vecinos de la Colonia } \\
\text { Zipor }\end{array}$ \\
\hline \multirow{3}{*}{ B } & $\begin{array}{l}\text { Gestión del taller en } \\
\text { la Subdirección de } \\
\text { Recursos Naturales y } \\
\text { Cambio Climático }\end{array}$ & - & Gestión & $\begin{array}{c}\text { Academia (Universidad } \\
\text { Veracruzana/MGAS) }\end{array}$ \\
\hline & $\begin{array}{c}\text { Compra de } \\
\text { herramientas para } \\
\text { los usuarios el } \\
\text { módulo }\end{array}$ & $\begin{array}{l}\text { Pala, bieldo, rastrillo y } \\
\text { regadera }\end{array}$ & Especie & $\begin{array}{c}\text { Academia (Universidad } \\
\text { Veracruzana/MGAS) }\end{array}$ \\
\hline & Impartición del taller & Residuos orgánicos & $\begin{array}{l}\text { Especie/ } \\
\text { Recursos } \\
\text { Humanos }\end{array}$ & $\begin{array}{c}\text { Departamento de } \\
\text { Agroecología y } \\
\text { Educación Ambiental }\end{array}$ \\
\hline
\end{tabular}

\section{Etapa 6: Diseño e Instalación de Huertos Comunitarios}

La etapa seis se integró por cuatro sesiones, consistió en el diseño e instalación de los huertos comunitarios. A través de estos huertos, los participantes vieron materializados los esfuerzos realizados durante la etapa anterior, ya que uno de sus propósitos fue utilizar el abono obtenido en los módulos de compostaje. En la tabla 3 se presentan las acciones correspondientes a las cuatro sesiones de esta etapa:

Tabla 3 Acciones, Insumos y Vinculaciones de la Etapa 6

\begin{tabular}{|c|c|c|c|c|}
\hline Sesión & Acción & Insumos & Tipo de Aportación & Vinculación \\
\hline \multirow[t]{2}{*}{$\mathrm{C}$} & $\begin{array}{c}\text { Planeación de la } \\
\text { sesión de diseño de } \\
\text { huerto }\end{array}$ & - & - & $\begin{array}{c}\text { Academia (Universidad } \\
\text { Veracruzana/MGAS) }\end{array}$ \\
\hline & $\begin{array}{l}\text { Sesión de diseño de } \\
\text { huerto }\end{array}$ & Papel bond y plumones & Especie & $\begin{array}{c}\text { Academia (Universidad } \\
\text { Veracruzana/MGAS) }\end{array}$ \\
\hline \multirow[t]{2}{*}{$\mathrm{D}$} & $\begin{array}{c}\text { Compra de } \\
\text { materiales para } \\
\text { germinar semillas y } \\
\text { para construcción de } \\
\text { huerto }\end{array}$ & $\begin{array}{l}\text { Semillas, plantas, } \\
\text { tepezil, composta, } \\
\text { tierra, semilleros, } \\
\text { tarimas de madera, } \\
\text { clavos }\end{array}$ & Especie & $\begin{array}{c}\text { Vecinos de la Colonia } \\
\text { Zipor/Departamento de } \\
\text { Agroecología y EA }\end{array}$ \\
\hline & $\begin{array}{l}\text { Sesión de } \\
\text { germinado de } \\
\text { semillas }\end{array}$ & $\begin{array}{l}\text { Semillas, tepezil, } \\
\text { composta, tierra, } \\
\text { semilleros }\end{array}$ & Especie & $\begin{array}{c}\text { Academia (Universidad } \\
\text { Veracruzana/MGAS) }\end{array}$ \\
\hline $\mathrm{E}$ & $\begin{array}{l}\text { Gestión de tequio } \\
\text { para instalación de } \\
\text { huerto }\end{array}$ & - & Gestión & $\begin{array}{c}\text { Academia (Universidad } \\
\text { Veracruzana/MGAS) }\end{array}$ \\
\hline
\end{tabular}




\begin{tabular}{cccc}
\hline $\begin{array}{c}\text { Instalación de } \\
\text { huerto }\end{array}$ & $\begin{array}{c}\text { Plantas, tierra, } \\
\text { composta, tepezil, } \\
\text { tarimas, } \\
\text { clavos/Herramienta }\end{array}$ & $\begin{array}{c}\text { Especie/Recursos } \\
\text { Humanos }\end{array}$ & $\begin{array}{c}\text { Red de Huertos Urbanos } \\
\text { y Periurbanos de } \\
\text { Xalapa/Vecinos de la } \\
\text { Colonia Zipor }\end{array}$ \\
\hline & Seguimiento y Evaluación \\
\hline Nota: elaboración propia.
\end{tabular}

\section{RESULTADOS Y DISCUSIÓN}

Para cumplir los objetivos de este trabajo se formaron dos grupos de vecinos que ocuparon las Áreas Verdes A y B. El Departamento de Agroecología y Educación Ambiental impartió dos talleres de capacitación para la separación de RSU y para el manejo del módulo de compostaje; se construyeron dos módulos. Para impartir la capacitación para la instalación de los huertos comunitarios, se contó con la colaboración de la Red de Huertos Urbanos y Periurbanos de Xalapa.

El compostaje ayuda a valorizar los sustratos orgánicos (Ruíz, 2009), convirtiéndolos en un área de oportunidad, mientras que el hecho de ser comunitario hace que el grado de particioación en el manejo integral de los residuos sea alto y directo (Martín \& Urquiaga, 2016). Por otro lado, los huertos comunitarios resaltan los valores ambientales y culturales, la recuperación de espacios urbanos de uso común y la socialización e intercambio de experiencias (Vaquerizo, 2015).

\section{CONCLUSIONES}

Los participantes de la Colonia Zipor se están apropiando del proyecto y están respondiendo de manera positiva a los cambios de hábitos que implica la separación de residuos y el compostaje. Actualmente nueve familias (en promedio cada familia tiene 4 integrantes) separan sus residuos en casa y ocupan los módulos de compostaje para el tratamiento de la fracción orgánica, cinco vecinos han creado un rol de supervisión para realizar periódicamente las actividades de mantenimiento y manejo de los módulos de compostaje.

La participación ciudadana, así como la vinculación con otros actores sociales como lo son las autoridades y la academia, fortalece la co-responsabilidad que el manejo integral de residuos implica y exige para ser llevado a cabo con éxito. La separación de residuos in situ, así como el tratamiento biológico de la fracción orgánica puede llevar a eficientar la recolección de estos y disminuir la cantidad que llega a los sitios de disposición final. 


\section{REFERENCIAS}

Aguilera, C. (2016). Estrategia de intervención comunitaria en torno a la implementación de una ecotecnia en la localidad "El Paisano”, Las Vigas de Ramírez, Veracruz (Trabajo recepcional). Maestría en Gestión Ambiental para la Sustentabilidad. Universidad Veracruzana. Xalapa, Ver.

Applus+. (2016). Propuesta definitiva de recogida de la fracción orgánica. España.

Hernández, C. (2014). Huertos familiares una estrategia para la sustentabilidad y seguridad alimentaria: aplicado en la comunidad de Santa María del Monte; Zinazantepec, Estado de México. Universidad Autónoma del Estado de México.

Martín, B., \& Urquiaga, R. (1 de marzo de 2016). Experiencias de compostaje comunitario en el Estado español. Ecologistas en acción(88). Obtenido de Ecologistas en acción.

Meza, D. (2010). Manejo sustentable de residuos a través de la elaboración de composta para la implementación de pantallas cortaviento en jardines residenciales en Nacozari y Esqueda, Sonora. Hermosillo: Universidad de Sonora.

Robles, M., Gasca, S., Quintanilla,. L., Guillén, F., \& Escofet, A. (2010). Educación Ambiental para el manejo de residuos sólidos: el caso del Distrito Federal, México. Investigación Ambiental, 2(1), 46-64.

Ruíz, F. (2009). Ingeniería del Compostaje. Chapingo: Universidad Autónoma de Chapingo.

Vaquerizo, A. (2015). Espacios verdes comunitarios: cómo potenciar la resiliencia urbana. Madrid: Universidad Autónoma de Madrid. 